III. Aus der Königlichen chirurgischen Klinik des Herrn Geheimrath Mikulicz in Breslau.

\title{
Zur Operation der grossen Chondrome des Rumpfes; ein Beitrag zur Chirurgie des Zwerchfelles.
}

Von Dr. W. v. Noorden, München, früherem I. Assist. der Klinik.

Von den an der Klinik des Herrn Geheimrath Mikulicz beobachteten und operirten Neubildungen nahmen im letzten Jahre zwei Fälle das Interesse besonders in Anspruch. - Beiden Gewächsen war eine ausserordentliche Grösse gemeinsam; histologisch gehörten sie ebenfalls derselben Gewebeklasse an, und beide gingen vom Skelett der grossen Rumpfhöhlen ans, um sich je in eine solche hineinzusenken. Die Operationen boten grosse Schwierigkeiten der anatomischen Lage wegen. Es handelte sich um gewaltige Tumoren der Thoraxwandung und des Beckenskeletts, die den Knorpelgeschwülsten und ihren Mischformen zuzurechnen waren.

Ueber den einen der Fälle hat Herr Geheimrath Mikulicz schon auf der 64. Naturforscherversammlung in Halle ${ }^{3}$ ) kurz berichtet. Bei dem Interesse, welches der Fall in Bezug auf die operative Technik bietet, ist eine ausführlichere Wiedergabe der Krankengeschichte gerechtfertigt.

I. Myxochondrosarkoma cysticum thoracis, Resection von vier Rippen, mit Entfernung eines handtellergrossen St ïckes des Zwerchfelles. Heilung.

Anamnese: Der 54 jährige Schmied J. ist in keiner Weise hereditär belastet, hat keine bemerkenswerthen Vorkrankheiten durchgemacht, auch keine Syphilis. Einer besonderen Verletzung der jetzt erkrankten Körperregion erinnert er sich nicht. Eine Beeinträchtigung des Allgemeinbefindens und hierdurch auch der Arbeitsfähigkeit hatte bis vor kurzem nicht statt. Beschwerden erwuchsen in letzter Zeit nur durch eine langsam zunehmende Schwellung an der linken Brustseite. Diese, nunmehr bei der Arbeit hindernd, fuhhrte den Patienten in die Klinik. Soweit der Kranke angeben kann, fühlte er vor vier Jahren schon eine htthnereigrosse derbe Anschwellung in der linken vorderen Axillarlinie in der Höhe der siebenten Rippe, welche nach und nach die bestehende Form gewann und seit kurzem schneller zunehmend die jetzige Ausdehnung erreichte. Während der untere Theil der Geschwulst immer unbeweglich war, haben sich die der Achselhöhle zugelegenen Partieen immer beweglich gezeigt und auch etwas weniger derb angefühlt. Von Schmerzen, Athembeschwerden war niemals die Rede.

Statuis praesens: Der Kranke bot folgendes Bild bei seiner Auf nahme am 25. Juni 1891. An der linken Thoraxseite sieht man eine gewaltige, im ganzen birnförmig gestaltete Geschwulst. Sie reicht von der Achselhöhle bis zum Thoraxende herab, uberschreitet nach vorı

1) Munzer, Prager medicinische Wochenschrift 1892 No. $34--35$.

2) Munzer l. c.

3) Siehe Verhandlungen Bd. II. S. 263. 
unterhalb der Brusțarze die vordere Axillarlinie. erstreckt sich rỉckwărts unterhalb des unteren Scapulawinkels in breiter Weise bis zum ausseren Rande der grossen Rilckenstreckmuskel und nahert sich hinten ulten bis auf $1 \mathrm{~cm}$ der Dalmbeinschaufel. Das Schulterblatt ist etwas nach oben verdrängt, doch beweglich. Der Umfallg der Geschwulst beträgt an seiner Basis gemessen $78 \mathrm{~cm}$, als Längenausdehnung in vertikaler Richtung elcreben sich $35 \mathrm{~cm}$. - Die Ausdehnung im anterior-posterior-Durchmesser beträgt in der Höhe der achten Rippe $26 \mathrm{~cm}$. - Nach anatomischen Regionen eingetheilt waren also befallen die Achselhöhle der linken Seite, die laterale Brustwand, Gebiete der Regio hypochondriaca und der Regio mammaria, endliclı unterhalb der Scapula liegende Theile des Ritckens. -

Die Athembewegung hat auf die Gestalt des Tumors keinen Einfluss; derselbe hebt und senkt sich mit del Thoraxhewegung. Die obele ExFig. it.

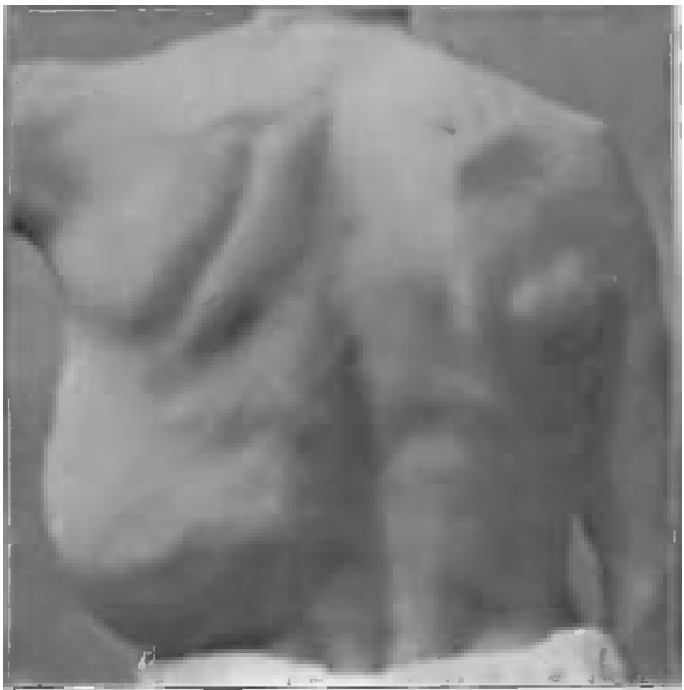

Fเป็. 2.

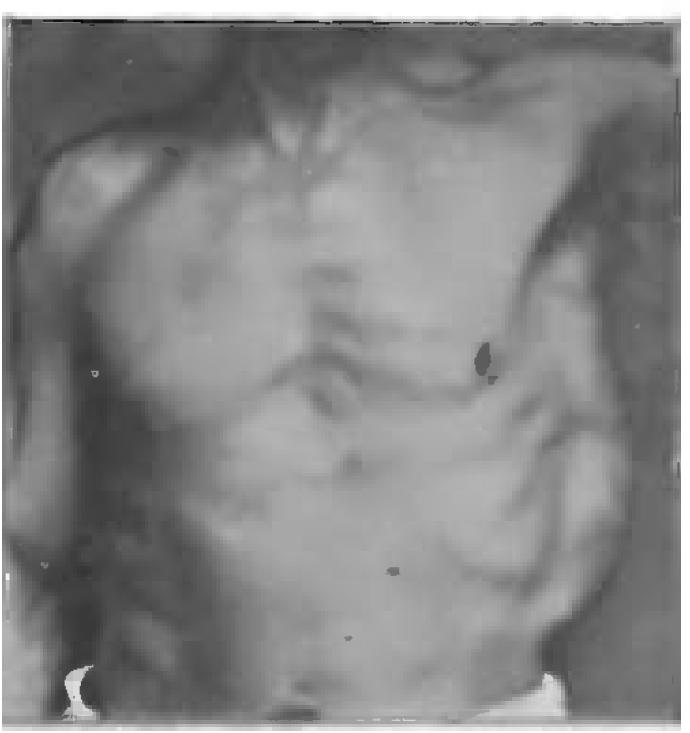

tremität ist in der Adduction entsplecllend bescllänkt, sie selbst flei voll nervösen oder Ciłculationsstörungell. - Keinerlei entzìndliche Erscheinungen sind im Bereich der Geschwulst wahrnehmbal. - Die Haut orweist sich zumeist als verschiebbar. und lier und da erscheint sie straffer gespannt und lässt zalte Gefässchen durchschimmeln. Durcl die Palpation ergiebt sich uberall gleiche Delbheit and Dichtigkeit der 'Tumolmasse, ausser in der Hohe der achten Rippe, wo eine Partie in der Tiefe vielleicht enveicht ist, doch kann mit der Spritze nichts aspirilt werden. Während zwei Drittel der Geschwulst eine einförmige compacte Masse ohne wesentliche Unebenheit bildet, besteht der zur Achselhöle hingehende Geschwulstheil aus knolligen Zugen. Dieser Theil ist bewegbar gegen den Thorax, whhrend der untere grössere kugelige mit den Rippen eins ist. Obwohl beide Theile des Tumors eng zusammengehören, so ist zwischen beiden eine ziemlich tiefe, abgreifbale und sichtbale Schnürfurche zu erkennen. Die Geschwulst ist durch Palpation allseits abgrenzbar. hier steiler, dort flacher sich verlaufend. Die Pel'cussion über del Geschwulst giebt durchaus leeren Schall. - Die auscultatorischen und percutorischen Verhältnisse der linken Tholaxeingeweide sind nachweisbar nicht verändert; speciell ist das Lungenathmen ausgesprochen vesiculär ohne Beigeräusche. - Sputum fehlt. Es liegt keine Herzverdlängung vor. An der Pleura kann ebenfalls nichts abnormes gefunden werden aucl fehlt ein pleuritisches Exsudat. Seitens der. Wirbelsäule und des tibrigen Skeletts und anderer Körpertheile, wie auch des gesammten Lynphdrtisensystems, liegen keine Verälderungen vor. Die,Bauchorgane sind normal gelagelt. Allgemeine und locale Staungserscheinungen fehlen. Im Urin fehlt Eiweiss, Zucker, Indican, man findet nur reichliches Sedimentum lateritiunl Der Hämoglobingehalt beträ̀t 85 (v. Fle ischl). Ein abnormer histologischer Bl utbef und kann nicht constatirt werden, man findet spälliche eosinophile Zellen, keine velmehrte weisse Blutkörperchen. Das Körpergewicht beträgt $68 \mathrm{~kg}$. Es ist ganz besonders hervolzuheben, dass die Athmung niclit alterirt war. Beide Thoraxhälften functionirten gleich krafiftig und ausgedehnt.

Die Diagnose derGeschwulst kollute aus clen anamnestischen Daten, dem localen und allgemeinen Befund uuter Zuhilfenahme einer vorhergehenden Probeexcision gestellt werden, obwoll diese mitunter nicht durehalls maassgebend ist, da ja oft bei Chondromen der Charakter der Geschwulst sicll nicht einheitlicl erweist. Carcinome, welche nicht von der Brustdrüse ausgehen, sind seltene Thoraxgeschwiilste und nelınen wohl niemals so gewaltige Dimensionen an, charakterisiren sich ausserdem bald durel Lymplidrüseninfection und Kachexie. Reine Sarkome, ob von den Weichtheilen oder dem Skelett ausgehend, liaben lasches Wachsthum: sie können dabei aucl ganz gewaltige Grössen erreiclien, ohne zu zerfallen, consumiren aber schnell die Kräfte. - Häufig fehlt jede Mitbetheiligung des Trympligefassgebietes. In ihrer Vorcesclichte spielt unerklärlicher Weise nicht seltell ein einmaliges lieftiges Trauma, eine Contusion, eine Rippenfractur ursäcliliclıp Rollen. Eminent chronisches Waclistlum - wie aucl lier seit vier Jalnen - zeigen die Chondroule. Osteome, und erst wenn sich Mischformen und Entaltungen bilden, beginnt schnelleres Waclisthunı. - Der Sitz, die lange Danel des Bestelıens, die geringe Beeinträclıtigung des Allgemeinbefindens, das Ausserspielstehen der Lymplibalınen und Lymplidrüsen, die Consistenz des Tuluor's und die fellende Hautbetleiligung liess Carcinon und Sarkonı differenzialdiagnostiscll ausscheiden und fül einen rutaltigen T'umor aus der: Bindegewebereilıe, für eiı Choudrom, vielleicht Myxochondrom entsclieiden, allerdings unit der Reserve, dass eine inaligne Entartuug vorlag wegen des kürzlich eilgetretenen sclınellen Waclistlum.s.

Eill werthvoller diagnostisclıer Wiık wurde durcl die Blutuntersuclung gewonnen. Die Hölıe des Hämoglobingehalts vou 80 bis $90 \mathrm{vor}^{\circ}$ der Operation entspricht nacl oft gemachten Erfalurulgen keinem maliguen Tumor. Andererseits ist es nun aucl wieder - wie sclıon lier bellerkt _. auf viele all unserer Klinik gewonlene Erfahrulgen gestützt, erlaubt, aus dem Wiederkehren des Hämoglobingelıaltes zur alten Hölıe eiıen Sclılıss darauf hin zu unaclien, dass vorläufig kein Recidiv eingetreten ist, odel wenigstens kein Recidiv maligner Natur.

Die Vorbereitung zur Operation bestand il den üblichen Maassnalımell, Fasten, Abfïlı'eı, Bad, und antiseptischer localer Vorbereitulg. Die Operation selbst wurcle nach den Principien der Asepsis ausgefülıt. Die Länge der Morplinunı-Chloroformnarkiose belief sicll auf annäher'ud 3/4 Stunden. Ein Zwischenfall fand nicht statt. Die Operation wurde il rechter Seitenlage des Kranken mit Erllölıung der Mitte des Thorax, wällrend der linke Arm stark elevirt wurde, ausgeführt. Die Operation verläuft in zwei Acten. Il der ersten Zeit ist die Pleura noch nicht eröffnet. Nach einem Lällgsschnitt über die Geschwulst und Freipräparation dieser von der iiberdeckenden Haut jederseits bis zur Basis wird der" 'Tumor" unter" peinlicher' Blutstillung in seinen bewegliclen oberen Tlieilen in Angriff genonmen. Die Jösung von der Achsellıöhle und Befreiung vou den begrenzenden Musculi pectoralis und latissimus dorsi und Langer'schen Acliselbogen gelingt anstandslos. Immerllin ist eine Lösung der del Tloraxwand schou lier breit aufsitzenden, aber niclit intin vel'wachsenen Gescliwulst von dell Rippen und $\mathrm{del}^{\circ}$ Intercostalmusculatul nothwendig. Je Illelır abwärts das Messer rordringt, desto inniger ist ler Tunior mit der 'Thoraxwand rerschmolzen; es erweist sicl, dass die siebente mit den viel folgenden Rippen und die Rippen-Zwischenmuskeln vollkonumen im Tumor aufgeangen sind, deingemäss von einel weiteren Isolirulg des Tumors abgesehen werden muss. -. Es werden deswegen zunächst nach vorn und linten die Hautdecken noch llellr abpräparist, und dann die Rippen in der linteren und rorderen Axillarlinie durch Abheben der Periostes zul Resection vorbereitet. - Hierbei erfolgt an einer kleinen Stelle die Pleuraverletzung. Der Pneumothorax hat jedoch auf das Allgenleilbefinden, Helz uld Atlimung keine Folgen, veranlasste aber zu schnellerem Vorgelıell, zumal die unbedingt lothwendige rechte Seitenlage während der Operation die Gefalir einer Atheminsuffienz erlöhten musste.

Ill sclineller Folge werden nunulehr d'ei Rippen sammit '/wischenuluskeln mit der Rippen-Knorpelscheer'e und der gebogenen Scheer'e durchschnitten. Da die Lunge woll auf ihr halbes Volumen sofort collabirt war, gelang dies leicht. Selbst bei den stärksten Iuspirationen trat die Lunge lange nicht in den Bereich des Operationsfeldes. - Die Blutung aus den Intercostalgefässen stillte vorläufig Compression mit aseptiscller Gaze, Bin Tlieil der 
Pleurahöhle wurde num vorsichtig mit sterilisirten trockenen Tüchern gepolstert. Bein Einblick in die Pleurahöhle zeigte sich, dass im unteren Raune die Verhaltnisse schwieriger wurden. Der Tumor mit den gelösten Rippen liess sich nicht nach auswärts schlagen, um abgetragen zu werden. Beim Zuge am Tumor wurde clas Zwerchfell gezerrt, welches in breiter Ausdehnung in die Geschwulst überging. - Eine Lösung vom Diaphragma durch Präparation erschien nicht thunlich, da dann für eine radikale Entfernung des Tumors nicht aufzukommen war; vielleicht wäre eine solche technisch in der grossen Tiefe ohne Perforation des Zwerchfelles auch gar niclit ausfüllrbar gewesen.

Herr Geheimrath Mikulicz entschloss sich deshalb sofort zur Resection des in Mitleidenschaft gezogenen Zwerchfelltheiles. Die Zwerchfellpartie, die dem Tumor hier als Basis diente, wurde mit der gebogenen Scheere herausgeschnitten, die starke Blutung sofort wit Klemmen beherrselit. Die Resection begann vorne, schritt zur Mitte, dann nach lintel mittels eines Bogenschnittes, indem überall mindestens ein anderthalb Centimeter freier Saum am Tumor verblieb. - Sofort nach der Ausschneidung des Zwerchfells konnte der grosse Tumor mühelos nach aussen gewälzt und nun leicht gelöst werden. - Nach dem Einschneiden in das Zwerchfell and während der Operation erwies sich als schwierig die Aufgabe des Zurückhaltens der unter starker Banchpresse stehenden Därme. Bei jedem Pressen drängten sich die Eingeweide in den Zwerchfelldefect und konnten nur mit grossen Tüchern gehalten werden. Die vordrängenden Darmschlingen gehörten hauptsächlich der Flexura coli an, aber auch der Magen, das grosse Netz und die Milz wurden sichtbar. Beim Einblick in die grosse Wundhöhle sieht man nocl einzelne Tumorknollen, scheinbar isolirt, in der Tiefe der Wunde gegen die Wirbelsäule hin lagern. Um diesen nachzugehen, war noch mehr Platz erforderlicl,, welcher durch weitere Entfernung von Rippentheilen nach hinten geschaffen werden konnte. Die vier Rippen fielen bis in die Gegend der hinteren Scapularlinie. Nunmehr war es unter Verdrängung der Lunge und der vorquellenden Bancheingeweide möglich, die direct neben der Wirbelsäule liegen den Tumorknollen mit Scheere, Pincette, Hammer und Meissel von den inneren Flächen der Rippenanfänge gründlich fortzunehmen.

War hiermit die Exstirpation des Tumors beendet, so begann jetzt die Versorgung der grossen Wundhöhle. Die erste Aufgabe war die Wiedervereinigung des Zwerclifelles. Der grosse Defect, etwas dreieckig gestaltet, von einem Tiefendurchmesser von $8 \mathrm{~cm}$ und nahe $9 \mathrm{~cm}$ Breitendurchmesser, konnte nicht durch einfaches Aneinanderlegen der Zwerchfellmuskelränder geschlossen werden, weil die Spannung zu stark war, und bei jeder forcirten Athmung und beim Pressen die Naht allzu gefährdet werden musste. Der Defect liess sich aber unter Zuhilfenahme der äusseren Thoraxwandung, speciell der breiten Bauchmuskel, schliessen. Nachdenı eil Theil des Zwerchfelles vorn und hinten direkt mit deu gelösten Bauchmuskehr vernäht war: konnte der Rest, eine $5-6 \mathrm{~cm}$ lange

$$
\text { Fig. } 3 .
$$

Fig. 4.
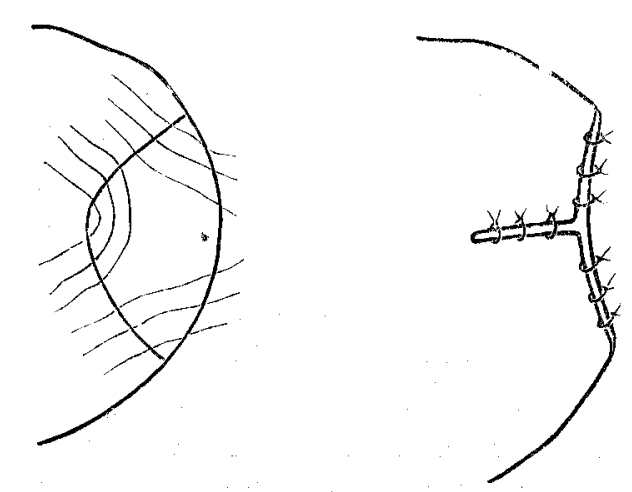

klaffende Spalte, ohne Spanmung nit einander in radiärer Richtung durch fortlaufende Naht vereinigt werden. Zum. Nalitnaterial diente feine Seide. Die beistehenden schematischen Abbildungen sollen den Gang der Zwerchfellnaht veranschaulichen. Fig. 3 stellt den. Verlauf der Nähte vor Knüpfung derselben, Fig. 4 nach vollendeter Nalit dar. Mit dem Schluss der doppelt angelegten Knopfnalit war ein sicherer Abschluss gegen die Bauchhöhle ge-

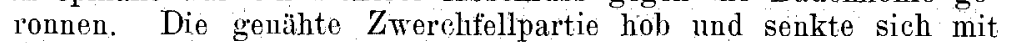
der Athimung anf und ab. Der Zwerchfellnalit folgte sorgfältige Austrocknung des Brustraumes mit aseptischen Tüchern und sorgfältige Versorgung der Blutgefässe, sodann ernente Tamponade. Hierauf wurde die änssere Wunde von der Achsel bis zur Thoraxbasis mit fortlaufender Seidennalit und tief in die Muskulatur greifenden Silberdrahtı̈̈liten gescllossen. Bevor dia letzte Oeffnung vernäht wurde, wurden die kurz vorher in die Brustliölle gelegten
Tücher herausgezogen. Die Trockenheit derselben bewies: die Blutstillung, so dass auch die letzte Oeffnung der Wunde verschlossen werden konnte. Der Nallt folgte eiı leicht comprimirender Jodoformgaze-Mooskissenverband.

Verlauf: Der Nachmittag und Abend verlief ohne Störung, ohne Eintritt bedrohlicher Erscheinungen. Da die Bauchhöhle eroffnet war. erschien es zweckmässig. Opium, nnd $1 \mathrm{~m}$ die Expectoration zu fördelm. Ipecuanhai infus zu verabreichen. Dieses wurde mehrere Tage fortgesetzt. Die Athmung, vor der Operation normal, erreichte eine Frequenz von 32-36 im Laufe des Abends und blieb danach meistens in den folgenden acht Tagen in dieser Hohe. Nach 72 Stunden stieg die Temperatur abends auf 39,2 , die Pulsfrequenz anf 132 ohne allgemeine Verschlechterung. Am folgenden Tage war die Abendtemperatur wieder 38,8, die Pulsfrequenz 124, den ganzen Tag uber 100, dabei die Dyspnoe etwas grösser. Von da bis zum achten Tage wurde keine bemerkenswerthe Storung und besorgnisserregende Erscheinnng im Allgemeinbefinden, besonders auch keine grosse Anänie bemerkt. Am achten Tage klagte der Patient morgens uber Druck auf der operirten Seite und tiber Athembescliwerden. Die Pulswelle ging hoher, etwas höher als in den letzten 'Tagen, der Puls war zugleich etwas unregelmässig. Peritoneale Reizerscheinungen waren aber nicht dabei. Dieser Befund veranlasst zum ersten Verbandswechsel. Man sieht links neben der Nahtlinie am Rippenbogen und in der Lumbalgegend eine kugelige, deutlich fluctuirende Vorwölbung. Jede entzlindliche Reaction an der Wundnuht fehlt. Die Percussion ergiebt schar abgegrenzte Dimpfung auf der ganzen Thoraxseite bis zul Mitte der Scapula. Durcll sofortige Probepunction war dickflussiges theerartige. Blut ohne mikroskopisch nachweisbare Beimischung von Eiter nachge wiesen. Hierauf wird durch Troikartpunction in der hinteren Axillarlinie circa $1 \frac{1}{2} 1$ dieser blutigen Flussigkeit entleert. - Mit Blut beschickt Nährböden bleiben steril. Nach der Entlastung wird der Atllemzug freier man sieht die des Skeletts beraubte Thoraxfläche bei der Athmung vol' und einwïrts wogen. Nach diesem Eingriffe wurde ein neuer leichter Jodoformgaze-Mooskissen-Compressionsverband angelegt.

In dem ganzen weiteren Verlaufe bis zill Entlassung ist nichts von Besonderheit hervorzuheben. Die Näthe wurden nach 14 Tagen entfernt Der Kranke erholte sich schnell, so dass er nach funf Wochen entlassen werden konnte und nach acht Wochen arbeitsfähig war. Schon nach 20 Tagen war die Athmung ganz ungestört. Icl will nocll erwähnem. dass der Hëmoglobingehalt (vor der Operation 85) bald nach der Operation 75 betrug. Am vierten 'Tage wurde ein grosser' Abfall anf 50 . nach drei Wochen anf 40 bemerkt; dieser niedrige Hämoglobingehalt hielt in der nächsten Woche an, doch wurde die Zahl 70 nach Monaten wieder erreicht Der scliroffe Abfall am Ende der ersten Woche nach der Operation mus: durch die Nachblutung gedeutet werden. Seit derOperation ist tiber ein Jahr vergangen, während dessen der Kranke dem Beruf als Schmied und Schlosser ununterbrochen nachgegangen ist und sich dauernd wohl befand. Vor einigen Monaten bemerkten wir am unteren Narbenende subcutan einige kleine larte Knollen, welche der Vorsicht wegen sofort entfernt wurden und sich in der That als reine Chondromgescliwilstchen erwiesen; sie waren entweder damals tibersehen und steheu geblieben. oder wir haben es mit einem Recidiv zu thın, doch spricht das ganze iibrige Verhalten, das Allgemein- und Localbefinden wenig fur ein Recidiv Durch einen kleinen Eingriff, der den Kranken wenige Tage an's Bett fesselte, wurden diese haselnussgrossen harten Geschwïlstchen excidirt

Der Status praesens bietet sich nach 14 Monaten folgendermaassen dar: Das Allgemeinbefinden ist gut. Klagen irgendwelcher $\Lambda_{1} \cdot t$ sind nicht vorhanden. Der Mann ist vollauf arbeitsfinhig. - Das Körpergewicht beträgt $65 \mathrm{~kg}$, der Hämoglobingellalt $65-80$ (v. Fle ischl). Der Blutbefund ist normal, keine Vermehrung der weissen Blutkörper, keine Vermehrung der eosinophilen Zellen, keine Drusenschwellungen. keine Stauungserscheinungen. - Urin normal, Stuhlgang normal. Thorax: Die Brust erscheint, von vorne gesehen, beiderseits gleiclımässig. Die linke obere Partie macht bei tiefen Inspirationen aber weniger ausgiebige Excursionen. Der Herzspitzenstoss ist nicht sichtbar, doch fulllbar im fünften Intercostalraume einwärts von der. Mammillarlinie. Die Herzdämpfung ist nicht verlagert oder vergrossert. Der Lungenschall ist vorne riberall hell und laut, kein Emphysem. Die Betrachtung des Thorax vom Ruicken ergiebt eine etwas geringere Atlimungsbewegming in dell unteren Purtieen. Deutlicher Lungenschall reicht bis zur neunten Rippe. Nan sieht auf der linken Seite eine starke Einziehıng des Thorax von der Höhe der acliten Rippe an nach abwärts. und zwar von der verlängerten medialen Scapuldarlinie an vorwärts bis in die Gegend der vorderen Axillarlinio reichend. Dieses Gebiet entspricht dem Defecte in der Thoraxwand. Bei jeder Exspirationsstellung des Thorax ver'wandelt sich die eingezogene Fläche in eine sich vorsttilpende, wulstige, gut fallstgrosse Wölbung. Der Schall ist hier gedämpft. Durch die Palpation constatirt man, dass das durcl die Athming veränderliche Thoraxwandgebiet kinöclier'n umrandet ist, und zwar nach oben und vorne durch die achte Rippe, nach hinten durch Rippenreste, deren Stïmpfe undeutlich durchfühlbar sind; vorn und seitlich begrenzen derbe Weichtheilmassen narbiger Natur den Defect. Das ganze Gebiet, dem die knöcherne Unterlage fehlt, lässt sichl eindr'tcken, so dass man bequem die ganze Vorderhand in den Defect rerbergen kann. Die Auscultation ergiebt uber dem ubrigen Thorax vesiculires Athmen, speciell sind uber und nahe dem Operationsgebiete keine abnormen auscultatorischen oder percutorischen Verhailtnisse zu finden; ich liebe hervor, dass wir anch stets bei den Nacluntersuchungen auf pleutitisches Reiben, wie es beim Vorkommen von Neubildungen auf der Pleura beschrieben wird, gefahndet haben. Die Wirbelsidule ist nicht skoliotisch verbogen.

Beschreibung 'des Tumors: Die makroskopische Betrachtung erlaubt den exstirpirten Tumor nit einer nugedreliten Birne zu vergleicheu. Seine Gestalt lidsst sich nach den Bildern 
im Tcxt leicht constrứren. Im unteren Theile sieht man die vier resecirten Rippen wenig mit dem Tumor verbunden. Sie selbst sind in der Continuität erhalten, demnach der Tumor wohl keinen centralen Knochenausgang hat. Die dem Tumor innen aufliegende Pleura glïnzt und ist hier und da'durch Knollen unregelmässig vorgestülpt; am unteren Ende des Tumor hängt fest mit ihm verwachsen ein über handtellergrosses Muskelsegment des $Z$ werchfells von $8 \mathrm{~cm}$ tiefer und $9 \mathrm{~cm}$ breiter Ausdehnung. - Ein weicher Muskelsaum ist frei von Tumor. Die Zwerchfellplatte ist nach unten nicht vom Tumor durchbohrt, wohl aber sehr verdünnt. Auf dem Durchschnitte wechseln dichte Partieen mit kleinen Hohlräumen. Knochen fehlt, aber Kalkeinlagerungen finden sich; mässig hartes Knorpelgewebe mit fasrigem Gewebe, an anderen Stellen mit sehr weichem Gewebe durchsetzt, bildet die Hauptmasse. Gewicht des Tumors 1000,0.

Mikroskopisch erhalten wir verschiedene Bilder. - An einigen Stellen wird Faserknorpel gesehen, anderswo nähert sich das Fasergewebe dem Schleimgewebe oder durch reicheres Auftreten von Rundzellengängen dem sarkomatösen Gewebe. Ganz besonders haben die der Achselhöhle zustrebenden Partieen diesen Charakter. Hiernach werden wir am besten die Geschwulst als eine Mischgeschwulst, und zwar als Myxo-chondro-sarkoma cysticum bezeichnen.

Fälle von Rippenresection mit Pleuraeröffnung wegen grosser Thoraxtumoren sind vielfach beschrieben; Fälle, in denen dabèi zugleich das Zwerchfell angetastet worden ist, sind spärlicher bekannt geworden.

Leisrink ${ }^{1}$ ) operirte im Gebiete der fünften und siebenten Rippe wegen eines Sarkoms. Dabei fand sich neben der Infection der Pleura auch ein Theil des Zwerchfelles durch einen etwa apfelgrossen Tumorrest ergriffen. Die Beseitigung verlangte eine circa fünfmarkstückgrosse Resection des Zwerchfelles. Auch in diesem Falle waren die prolabirenden Baucheingeweide (Leber, Darm) schwer zurückhaltbar, es gelang aber, die Zwerchfellwunde durch mehrere Nähte wieder zu schliessen. Der Fall endete durch Capillarbronchitis nach fünf Tagen tödtlich. Die Section erwies, dass die Zwerchfellnaht gehalten hatte und noch verstärkt war durch Adhäsionen zwischen der Nahtstelle und der Leberoberfläche.

Humbert ${ }^{2}$ ) referirt über einen Fall, in dem bei der Nachoperation wegen Thoraxsarkom das $Z$ werchfell verletzt wurde. Bei der Entfernung der noch Tumormasse tragenden neunten Rippe entstand ein Defect von $7 \mathrm{~cm}$ Länge und $2 \mathrm{~cm}$ Breite. Der Schluss der Zwerchfellwunde gelang durch fünf Catgutsuturen, der Patient lebte noch zwei Monate. In einem Falle, dessen Vorgeschichte ich Gelegenheit hatte, als Assistenzarzt bei Prof.. Bruns mitzubeobachten, resecirte $\mathrm{Hahn}^{3}$ ) später wegen Recidiv die sechste bis zehnte Rippe. Der recidivirende Tumor war sowohl auf die Pleura als auf das Peritoneum übergegangen. Nachdem ein ausserst grosser Eingriff zur Beseitigung des Tumors aus der Pleurahöhle und vom Bauchfell gemacht war, wurde das Zwerchfell an den oberen Wundrand angenäht, um Pleura und Abdomen gegen einander abzuschliessen. Der Fall endete tödtlich. Die Erfahrungen über Operationen am $\mathrm{Zwerchfell} \mathrm{hat} \mathrm{P}_{0}$ s te mpski${ }^{4}$ ) in neuester Zeit dankenswerth vermehrt und manche Eingriffe mit Glück durchgeführt. Für manche Fälle. nimmt er nach einer von ihm angegebenen $\mathrm{Me}-$ thode einen plastischen Thoraxlappen zur Hülfe.

Unser Patient hat seine Genesung zunächst vor allem dem zu verdanken, dass es gelungen ist, ihn während und nach der eingreifenden Operation über einen Collaps hinwegzubringen, dann aber dem, dass die Wundheilung vollkommen aseptisch verlief. Man mag sich auf den Standpunkt stellen und sagen: das ist selbstverständlich und nicht des Erhebens der Mühe werth, aber nur zu leicht kann bei Operationen, die eine solche Ausdehnung annehmen, so leicht inficirbare Höhlen und Flächen freilegen, die weiter einen grossen Apparat und zahlreiche Assistenz verlangen und lange Zeit beanspruchen, ein Fehler mit unterlaufen.

Die Durchführung der Asepsis mit voller Ausscheidung wirksamer Antiseptica ist in solchen Fällen aber ganz besonders geboten wegen der Gefahr der Resorption von der Pleura, dem Peritoneum und den Zwerchfellmuskeln selbst. Das Verhalten der Gewebe ist ein ganz anderes als da, wo durch chronische entzündliche Vorgänge eine verdichtete Pleura Schutz verleiht,

Die direkte Bespülung der zarten Lungenpleura mit Lösungen irgend welcher Art erscheint uns auch deswegen entschieden gefährlich, weil nicht abzusehen ist, in welcher Weise das Lungengewebe den thermischen und chemischen Reiz beantwortet. Nicht

1) Leisrink, v. Langenbeck's 'Archiv Bd. 26, p. 946.

2) Humbert, Revue de Chirurgie 1886 ; No. 4 .

3 Hahn; Deutsche medic. Wochenschrift 1888, No. 50.

4) Postempski, Verhandlungen des X. internationalen Congresses Bd. 3, p. 188 . unmöglich ist die Entstehung einer gefährlichen Bronchitis oder Pneumonie. Kolaczekl) berichtet, dass in einem von Fischer in der Breslauer Klinik operirten Falle die Lunge auf Ausspülung der Höhle mit Salicylsäurelösung mit eitriger Bronchitis geantwortet habe.

Auf Stillung der Blutung wird in Zukunft noch mehr Gewicht zu legen sein. Die ausserordentliche Nachblutung hätte leicht einen Strich durch die Rechnung machen können. Wahrscheinlich ist ein thrombosirtes Gefäss wieder zur Blutung gekommen, oder es hat Lockerung einer Ligatur stattgefunden. Wir nehmen an, dass ein mittelgrosses Gefäss, vielleicht eine Art Intercostalis intermittirend nachgeblutet hat. Dass die starke Füllung des Pleuraraumes mit Blut keine ängstlichen Symptome hervorrief, kann nur durch die Annahme einer sehr langsamen Blutung erklärt werden, oder dadurch, dass die linke Lunge, welche durch den Pneumothorax bereits ausser Athmung gesetzt war, durch die Anfüllung des Pleuraraumes nicht mehr berührt werden konnte, da ja sowie so die rechte Lunge vorläufig die ganze Athmung allein besorgte.

Die Eröffnung der Pleurahöhle bei der Operation der Thoráxtumoren durch weite Rippenresection ist an sich schon ein grosser Eingriff. Die Gefahr liegt in dem plötzlich auf dem Operationstisch auftretenden Pneumothorax mit vollkommenem Lungencollaps. Wie sehr die Lunge, und mit welcher Geschwindigkeit dieselbe collabiren kann, konnten wir sehr deutlich beobachten, da keinerlei pleuritische Verwachsungen bestanden, durch welche irgend welche Lungengebiete gehalten wurden, und durch welche die Lunge zum Theil wenigstens den Excursionen der Thoraxwand hätte folgen müssen.

Immerhin ist es interessant mitzutheilen, dass weder in dem Athmungsmodus noch in der Herzthätigkeit bemerkenswerthe Veränderungen während der Operation auftraten. Leider konnte wegen des nothwendigen Verbandes das Verschwinden des Pneumothorax nicht genau verfolgt werden; es sei aber bemerkt, dass schon nach drei Wochen die Athemfrequenz durchschnittlich 18 pro Minute betrug, und dass nach fünf Wochen heller Lungenschall bis vier querfingerbreit unterhalb der Spina scapulae nachweisbar war.

Die Betheiligung des $Z$ werchfelles war in diesem Falle nicht vorauszusehen, es wies ja kein Symptom darauf hin. Jederlei Abnormitäten im Athmungsmodus fehlten, auch chronischer oder paroxysmatisch auftretender Singultus. Im ganzen wird bei Tumoren, welche die unteren fünf bis sechs Rippen ergriffen haben, immer der Gedanke an Zwerchfellbetheiligung auftauchen, und man wird eine Erwägung abhalten müssen, ob man bei solcher Wahrscheinlichkeit zur Operation schreiten soll. Carcinome, Sarkome weicherer Form können, wie überall bei grösserer Ausbreitung, so auch hier wenig Aussicht auf Beseitigung versprechen. Osteome, Chondrome, Myxochondrome, oder dem Sarkomgewebe nahestehende Chondrome dürfen eher Hoffnungen erwecken - bezüglich ihrer Abgrenzung, wie in unserem. Falle. Sobald das Zwerchfell mit Wahrscheinlichkeit ergriffen ist, tritt weiter die Frage auf, ob die benachbarten Organe, die Leber resp. die Milz und Nierenkapsel auch das Peritoneum irgendwie Beziehungen zum Tumor haben, und dadurch eine Operation andere Gesichtspunkte gewinnt.

(Schluss folgt.) 